\section{Combined Confocal Imaging Reveals Interesting Complimentary Information}

Barbara Foster, Microscopy/Microscopy Education

Recently, a question surfaced on the Microscopy list server regarding preparation of insect eggs for EM evaluation. While the electron microscopy approach involved considerable sample preparation (mounting, coating, etc.), our experience indicated that, for some of these applications, a combination of reflected and fluorescent confocal microscopy provided valuable information with little or no sample preparation required, provided there are not other considerations such as the extended resolution provided by electron microscopy.

In the biological arena, most confocal work is done in fluorescence mode. There have been some forays into imaging silver-stained structures using reflected light confocal but those applications have not been very prevalent in the literature. With the judicious addition of a quarter wave plate (a polarizing accessory) in the light path, the disruptive artifact from the laser beam can be removed from the image, converting any confocal into a dual purpose instrument.

Figures 1 and 2 show the fluorescent and reflected light confocal images of a moth egg. Both were created using a Sarastro Phoibus Laser Scanning Confocal Microscope (LSCM). Thitty-one image slices, $512 \times 512$ pixels each, were taken at 10 micron steps, using a $10 \times / 0.45 \mathrm{NA}$ objective. No unusual sample preparation was used: the egg was just left on the plastic wrap to which it was anchored (the cause of the bright background reflection in Figure 2) and imaged. While no data is available on the wavelength used for the fluorescence, the laser was a standard argon ion model and the dichroics were most likely set to optimize for the 488 and/or $512 \mathrm{~nm}$ lines. The embryo and surrounding structures naturally autofluoresce in this region.
Once the images were collected, they were processed using integrated 3D soft-⿳亠丷厂犬 ware'.

Figure 1 is half of a stereo pair, showing the egg as imaged using fluores:cence. (Unfortunately, its partner was not available for publication). When seen $\overrightarrow{-}$ together, the pair show a highly three dimensional view of the developing embryor and egg sac within the egg. Figure 2 shows the external structure of the egg. imaged in reflected light. It provides exact registration and context for the inter nal view. Since the procedure is non-invasive and non-destructive, this com bined confocal technique can be used for tracing embryological developmento over time.

Although this particular specimen is biological in nature, any specimer which has internal structures which fluoresce and external structures which res flect is a likely candidate for this type of analysis. The switch from one imagin mode is readily accomplished by adjusting a few optical components (essentially filters), making the combined technique accessible even to novice users.

A word about the author:

Ms. Foster heads a consortium of microscopy experts who specialize in custom ized, on-site courses in all areas of microscopy, image analysis, and samplot. preparation. She is also the author of the recently released Optimizing Light Mic croscopy for Biological and Clinical Laboratories. To learn more about her comm pany and the book, visit their website: MME-Microscopy.com/education.

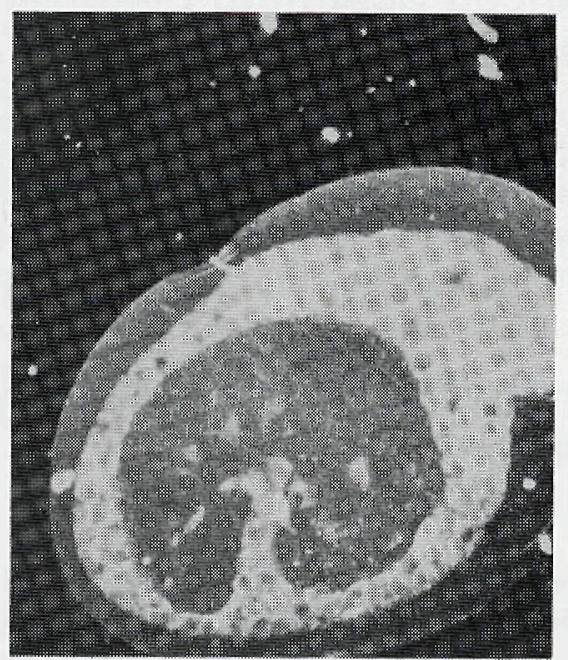

Figure 1: 3D reconstruction of fluorescent confocal image of a moth egg (Apoda biguttata).

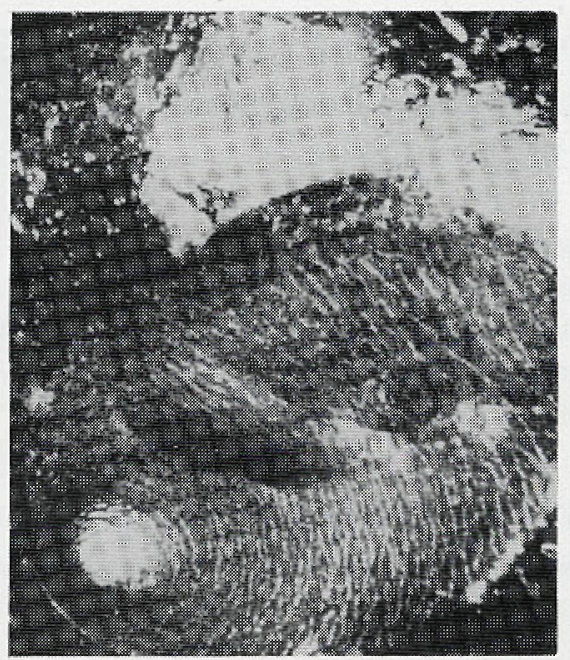

Figure 2: 3D reconstruction of the same egg, imaged using reflected light confocal microscopy.

Sample: courtesy of Dr. Marc Epstein (located at the Smithsonian Institute at the time these images were collected). Images: courtesy of Barbara Foster 
